\title{
Direct Determination of a Bare Neutron Counter Yield
} Function

\section{W. Nuntiyakul, ${ }^{a, b, *}$ P.-S. Mangeard,${ }^{c}$ D. Ruffolo, ${ }^{d}$ P. Evenson, ${ }^{c}$ J. W. Bieber, ${ }^{c}$ J. Clem, ${ }^{c}$} A. Hallgren, ${ }^{e}$ J. Madsen, ${ }^{f}$ R. Pyle, ${ }^{g}$ A. Sáiz ${ }^{d}$ and S. Tilav ${ }^{c}$

${ }^{a}$ Department of Physics and Materials Science, Faculty of Science, Chiang Mai University, Chiang Mai 50200, Thailand

${ }^{b}$ National Astronomical Research Institute of Thailand (NARIT), Chiang Mai 50180, Thailand

${ }^{c}$ Department of Physics and Astronomy, University of Delaware, Newark, DE 19716, USA

${ }^{d}$ Department of Physics, Faculty of Science, Mahidol University, Bangkok 10400, Thailand

${ }^{e}$ Uppsala University, Box 516, S-75120 Uppsala, Sweden

${ }^{f}$ Wisconsin IceCube Particle Astrophysics Center, University of Wisconsin-Madison, Madison, WI 53703, USA

${ }^{g}$ Pyle Consulting Group, Inc., St. Charles, IL 60174, USA

E-mail: waraporn.n@cmu.ac.th, psmangeard@gmail.com,

david.ruf@mahidol.ac.th, evenson@udel.edu, jwbieber@bartol.udel.edu,

clem@bartol.udel.edu, allan.hallgren@physics.uu.se,

jim.madsen@icecube.wisc.edu, RogerPyle@gmail.com,

alejandro.sai@mahidol.ac.th, tilav@bartol.udel.edu

Ground based neutron counters are a standard tool for detecting atmospheric showers from GeVrange primary cosmic rays of either solar or galactic origin. Bare neutron counters, a type of lead-free neutron monitor, function much like standard neutron monitors but have different yield functions primarily because they are more sensitive to neutrons of lower energy. When operated together with standard monitors the different yield functions allow estimates to be made of the energy spectrum of galactic or solar particles. In 2010 a new array of twelve bare neutron detectors was installed at the South Pole to operate together with the neutron monitor there. Prior to installation, two of the detectors were operated on a ship that traveled from Sweden to Antarctica and back from November 2009 to April 2010. The purpose of this latitude survey was to use Earth's magnetic field as a spectrometer, blocking cosmic rays below the local cutoff rigidity (momentum per unit charge), from which we determined the response function vs. rigidity of these bare counters. By comparing that measured response function to direct measurements of the cosmic ray spectrum taken by the PAMELA spacecraft, we were able to make a direct determination of the yield function for these detectors.

$37^{\text {th }}$ International Cosmic Ray Conference (ICRC 2021)

July 12th-23rd, 2021

Online - Berlin, Germany

\footnotetext{
${ }^{*}$ Presenter
} 


\section{Introduction}

A standard neutron monitor (NM64) [1] provides a ground based measurement of the flux of cosmic ray particles in space by counting neutrons present in the atmospheric cascade of secondary particles produced by the primary cosmic rays. Bare neutron detectors, a type of lead-free neutron monitor, are more sensitive to lower energy primary particles than a NM64 but are more sensitive to environmental effects $[2,3]$. As there is no standard design for a bare neutron detector each installation must be analyzed and calibrated individually since the performance may vary with construction technique.

The relation between the primary flux and the detected particles is most fundamentally expressed by the yield function $(Y F)$, which is an energy dependent effective geometric factor [4]. The differential response function $(D R F)$ is the product of the yield function and the primary particle spectrum. The counting rate of the detector is the integral over rigidity of the $D R F$.

The $D R F$ of a ground based detector can be measured by using the Earth's magnetic field as a magnet spectrometer. At any given location the magnetic field blocks cosmic rays below a certain cutoff rigidity $\left(P_{\mathrm{c}}\right)$, where rigidity is particle momentum per unit charge $(P=p c / q)$. In a latitude survey the detector is transported through a range of magnetic latitudes $[2,5,6]$ so that the counting rate can be measured as a function of geomagnetic cutoff rigidity. At each location of the survey the cutoff can be calculated from geomagnetic field models. The cutoff is also a function of time because the geomagnetic field is variable, both intrinsically and in response to the influence of the sun on the magnetosphere. Comparing two points with closely spaced cutoffs, the difference in counting rate divided by the difference in cutoff rigidity is exactly the $D R F$.

The present work reports data from a ship-borne latitude survey using two bare neutron counters during 2009 - 2010. The two bare neutron detectors were subsequently installed at the South Pole, along with ten unmoderated bare neutron detectors. To our knowledge this is the first report of a latitude survey of bare neutron counters that were also installed at a fixed station for long term measurements of cosmic rays, and also the first direct determination of a bare neutron counter yield function. During the survey there were no GeV-range solar particle events so the bare counters detected showers exclusively from galactic cosmic rays. Previous latitude surveys with bare counters were conducted with a single bare counter in 1976 [7], three bare counters in 1995-6 [3], and two bare counters in 1996 [2]. Each survey used a different bare counter design. The resulting DRF were compared by [3].

\section{Observation}

\subsection{Instrumentation}

The bare neutron detector design used in the latitude survey is shown in Figure 1. It consists of an LND25373 ${ }^{3} \mathrm{He}$ neutron counter [8] surrounded by a paraffin wax moderator inside a wooden box. Two such detectors were strapped on top of an ice Cherenkov detector of cosmic ray showers for the IceTop surface component of the IceCube Neutrino Observatory at the South Pole [9]. The detectors were housed in a temperature-controlled freezer container. This shipping container was then loaded onto the Oden icebreaker. 


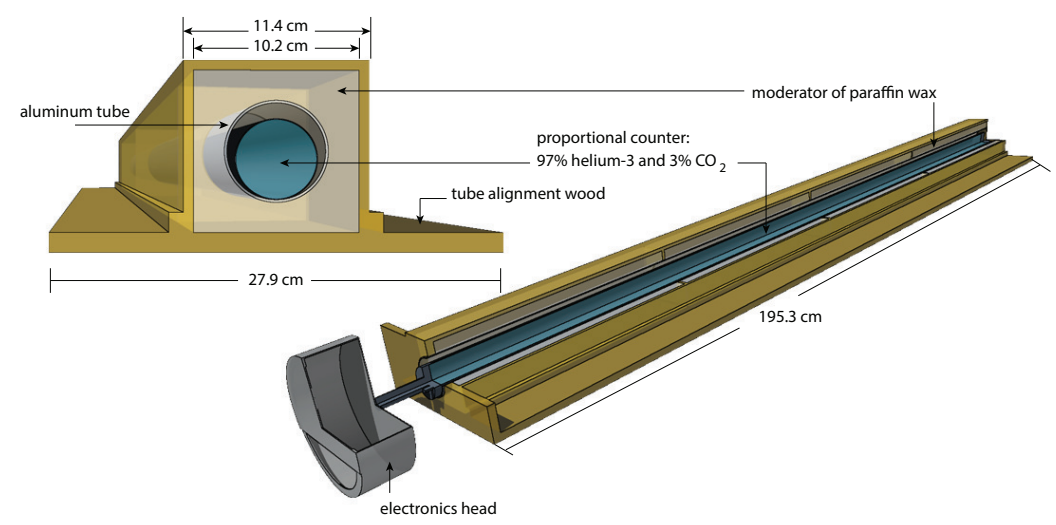

Figure 1: Rendering of an end view and cutaway oblique view of a paraffin moderated bare neutron counter to detect cosmic ray showers. The present work analyzes data from two such bare neutron counters operated on a ship for a latitude survey in 2009-2010. Since 2010 the two counters have taken data at the South Pole.

\subsection{The 2009 - 2010 Latitude Survey}

This survey year began on 11 November of 2009 from Helsingborg, Sweden at a cutoff rigidity of $1.88 \mathrm{GV}$. There was an extensive port call in Uruguay at approximately $8.2 \mathrm{GV}$, after which the survey continued to McMurdo Station, Antarctica. Note that in Earth's polar regions the geomagnetic cutoff rigidity can be quite low, but the primary cosmic ray rigidity must exceed an atmospheric cutoff of approximately $1 \mathrm{GV}$ for the atmospheric shower to generate counts in a ground level neutron detector. The return trip was via Punta Arenas at $5 \mathrm{GV}$, and Uruguay at $8.2 \mathrm{GV}$, and finally back to Sweden on 26 April 2010.

\section{Data}

Data were obtained with the standard acquisition system used in previous surveys [6]. The counting rate of each detector was recorded once per second together with the attitude of the ship (pitch and roll). Once per minute the barometric pressure was recorded, as was the position of the ship derived from GPS data, which also provided absolute timing. Apparent geomagnetic cutoffs [10-12] were calculated at half hour intervals.

As noted below, some additional intervals were also excluded from the analysis. Systematic corrections were only applied for barometric pressure and short term solar modulation of the galactic cosmic ray flux. A summary of the data is presented in Figure 2, while the exclusions and corrections are discussed below.

\subsection{Data Exclusions}

As in our previous bare neutron detector survey [3] and in the 1996 survey [2], the detectors showed major increases of the count rate ratio of the bare neutron detectors to the neutron monitor whenever the ship was in port. We removed those time periods from our analysis and also removed time periods with a clear effect of proximity to land near Göteborg Sweden, in the estuary of the river Plate in Uruguay, and in the Magellan Strait. We also excluded all of the data in the vicinity of McMurdo Station. Exclusions for these geographic reasons are indicated in blue in Figure 2. 

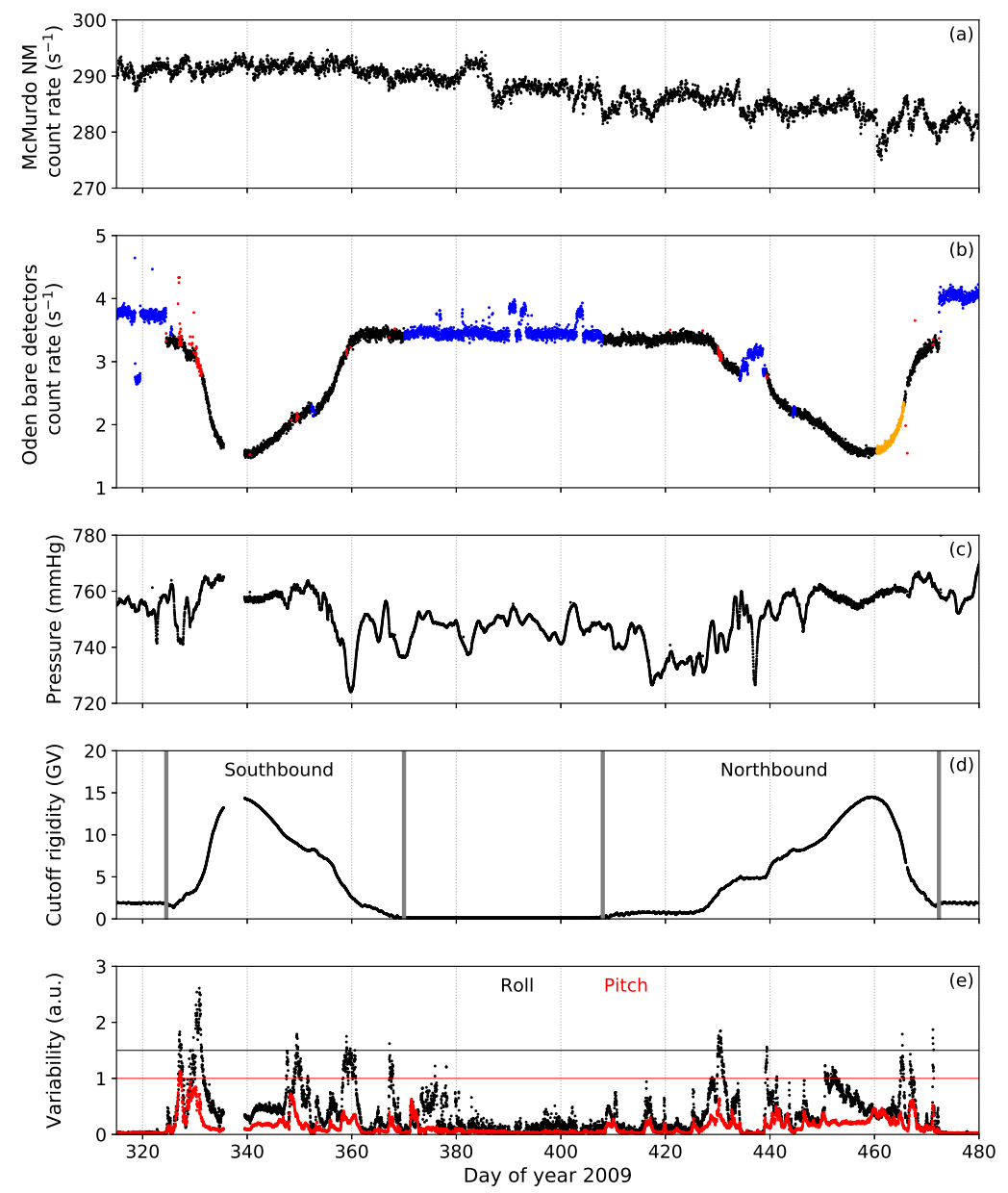

Figure 2: Data set used in this analysis. (a) Pressure corrected count rate of the 18NM64 McMurdo neutron monitor. (b) Pressure corrected average count rate of the two bare neutron counters on board the Oden. Black points were accepted as valid data while points of other colors were excluded. (c) Atmospheric pressure at the Oden in mmHg. (d) Apparent cutoff rigidity at the Oden in GV; vertical lines define the Southbound and Northbound intervals. (e) Standard deviation of Oden roll (black) and pitch (red) angles (arbitrary units). Horizontal lines show the applied cuts for rejecting time periods of strong pitch or roll.

Although we could detect no overall dependence of the counting rate on the attitude of the ship, there were indications of excess fluctuations related to high seas during some storm periods. We therefore excluded data when the standard deviation of the roll and/or pitch angles exceeded some (rather arbitrary) limits, shown in red in Figure 2. Also shown in red are a few points excluded due to obviously wrong counting rates or bad pressure measurements.

A major Forbush decrease due to a CME occurred during the northbound period. In terms of the fractional day of the year 2009 (DOY), we removed the data from $D O Y=460.433$ to $D O Y=465.641$ from our analysis to exclude the time period when the spectrum of cosmic rays at the top of the atmosphere was affected by this solar storm, including the recovery period. Data excluded for this reason are shown in orange in Figure 2(b). 
Table 1: Derived Parameters for Survey Intervals

\begin{tabular}{l|c|c|c|c|c}
\hline Parameter & $S_{0}$ & $S_{1}$ & $S_{2}$ & $S_{3}$ & $\bar{m}\left(\mathrm{~s}^{-1}\right)$ \\
\hline Southbound & $0.0130(2)$ & $0.0048(1)$ & $7(1)$ & $0.33(2)$ & 291.4 \\
Northbound & $0.0125(1)$ & $0.0052(1)$ & $12(2)$ & $0.38(2)$ & 284.8 \\
\hline
\end{tabular}

\subsection{Barometric Pressure Correction}

A strong anti-correlation is observed between a neutron monitor count rate and barometric pressure. For a typical latitude survey the detector does not spend long enough at each value of the cutoff (even when grouped into, for example, $2 \mathrm{GV}$ bins) to determine a pressure coefficient by the phenomenological method. By using 14 surveys with substantially equivalent instruments [6] were able to establish (for an NM64) the pressure coefficient, $\beta$

$$
\begin{gathered}
\beta=1.006 \times 10^{-2}-1.53 \times 10^{-4} P_{\mathrm{c}} \\
C_{p}=C e^{\beta\left(p-p_{\text {ref }}\right)}
\end{gathered}
$$

where $C_{p}$ is the pressure corrected counting rate, $C$ is the uncorrected counting rate, and $p$ is the barometric pressure in units of $\mathrm{mmHg}$, and $p_{\text {ref }}=760 \mathrm{mmHg}$.

\subsection{Short Term Modulation}

The McMurdo monitor count rate in Figure 2(a) indicates time variations in the galactic cosmic ray flux due to solar activity, known as solar modulation. Even a cursory examination reveals that the overall level of solar modulation is quite different near the early "southbound" and the late "northbound" portions of the survey. We consider this difference quite an important one to preserve in the analysis, and indeed think that a self consistent handling of this difference strengthens our determination of the yield function. Our correction for short-term variations in modulation level is based on variations in the McMurdo count rate. There are two steps to this analysis. First, following the methods of [6], we determined regression coefficients $(S)$ between the bare neutron detector count rate corrected for pressure for each rigidity bin, i.e., $0-1 \mathrm{GV}, 1-2 \mathrm{GV}, \ldots$, and $13-14 \mathrm{GV}$ and the McMurdo count rate corrected for pressure, $C_{P}$. Second, we found that the following function, with the parameters $S_{i}$ given in Table 1, provides a reasonably good fit to the data:

$$
S\left(P_{\mathrm{c}}\right)=S_{0}+\frac{S_{1}-S_{0}}{1+S_{2} e^{-S_{3} P_{\mathrm{c}}}}
$$

We then used $S$ calculated by equation 3 as a function of $P_{\mathrm{c}}$ to correct the counting rate to the average McMurdo value for each part of the survey separately:

$$
C_{p s}=C_{p}-S\left(P_{\mathrm{c}}\right)(m-\bar{m})
$$

where $C_{p s}$ is the normalized Oden neutron monitor counting rate corrected for pressure and short-term GCR variation, $m$ is the McMurdo counting rate, and $\bar{m}$ is the average McMurdo counting rate for the specific interval of the survey. $C_{p s}$ was used to determine the response function. 

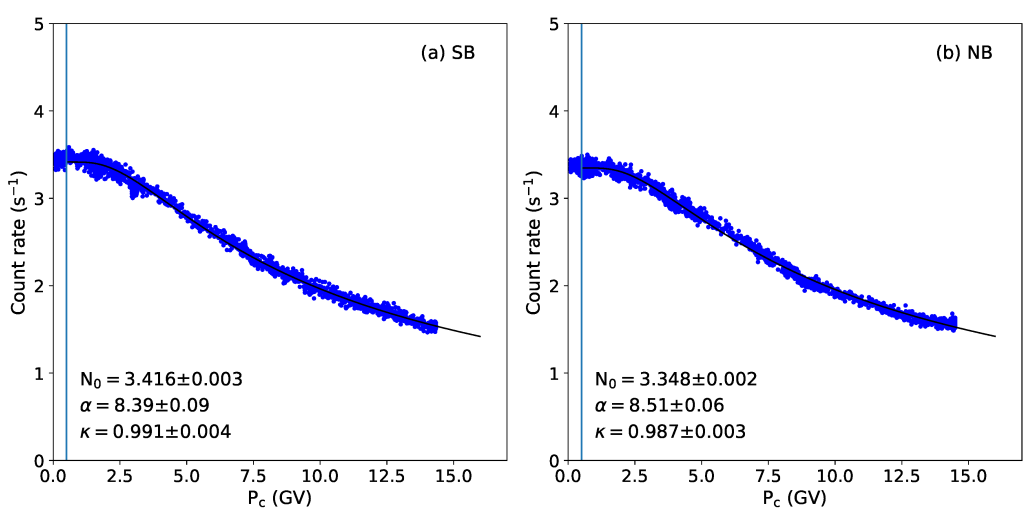

Figure 3: Separate Dorman function fits to (a) southbound (SB) and (b) northbound (NB) bare neutron detector count rates. The vertical line shows the lower cutoff limit used during the fitting procedure.

\section{Bare Neutron Detector Yield Function}

\subsection{Latitude Survey Response Functions}

Solar modulation conditions were different for the southbound and northbound intervals of the latitude survey. Figure 3 shows the Dorman function fit to the southbound and northbound intervals separately. In performing these fits we excluded all data from geomagnetic cutoffs below $0.5 \mathrm{GV}$.

\subsection{Direct Determination of Yield Functions}

We derived the yield function using an approach similar to that of [13] for a standard NM64. Conceptually the yield function of the neutron detectors is simply the ratio of the $D R F$ to the particle spectrum at the top of the atmosphere. A complication is that each particle species has its own yield function and spectra of different elements have different rigidity dependence. We approximate the multiple ion species actually present by assuming that there are only protons and ${ }^{4} \mathrm{He}$ nuclei (alpha particles), since the spectra of the heavier elements tend to resemble the alpha spectrum but at significantly lower intensity, and multiply the alpha spectrum by a scale factor $F_{h n}$ to account for heavier elements.

To evaluate the yield function several assumptions are made. First, we use the Local Interstellar Spectrum (LIS) derived from spacecraft and balloon data [14, 15]. A force-field approximation [17] of the solar modulation was applied to the LIS using the solar modulation parameter $\Phi$ determined by [16] for the time of interest. Then, we assume a ratio between the yield functions of protons and alphas, $F_{Y}(R)$, using an approach similar to that for a standard NM64 as proposed by [13]. Finally, we include the contribution of heavy nuclei by a multiplicative scale factor, $F_{h n}$, to the alpha particle yield function. We consider two published estimates of this scale factor. One is $F_{h n, C}=1.584$ from [13], to be consistent with their calculation of $F_{Y}$, and the second is $F_{h n, G}=1.445$ from [16] to be consistent with their calculation of $\Phi$. The yield function of the bare counters to protons is calculated as:

$$
Y_{p}(P)=\frac{D R F(P)}{J_{p}(P, \Phi)+F_{h n} F_{Y}(P) J_{\alpha}(P, \Phi)},
$$



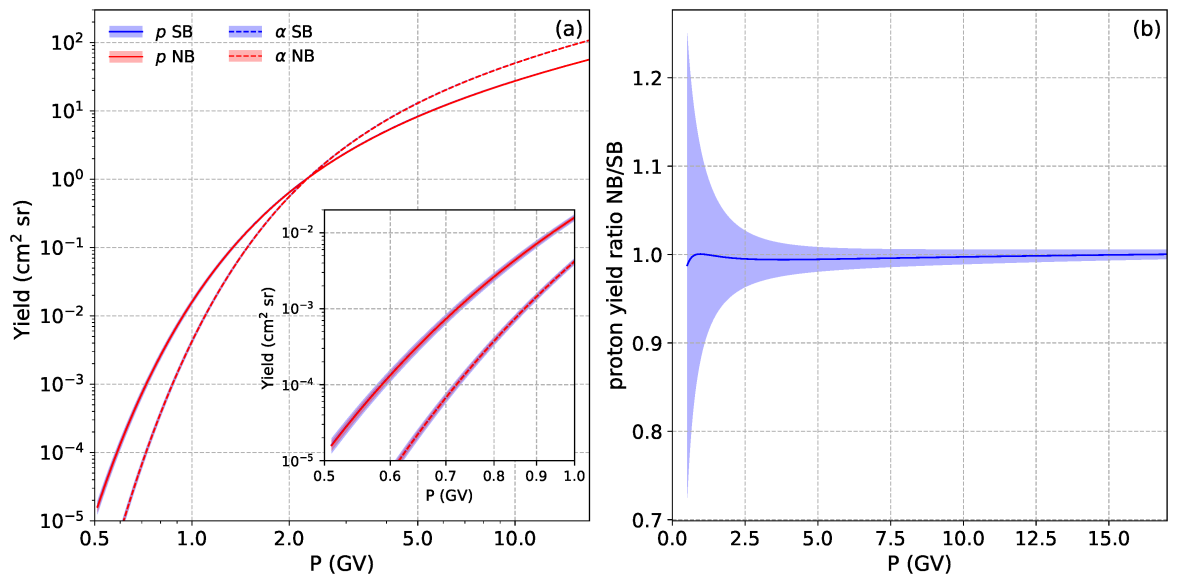

Figure 4: (a) Yield functions of the two bare neutron counters for protons and alphas derived from the 2009 - 2010 latitude survey and particle spectra at the top of the atmosphere as described in the text. (b) Ratio of the southbound to the northbound proton yield functions. We use $F_{h n, G}$ as the scaling factor from [16].

where $J_{p}(P, \Phi)$ and $J_{\alpha}(P, \Phi)$ are the spectra of protons and alphas at the top of the atmosphere, respectively. The yield function to the alphas is then determined as:

$$
Y_{\alpha}(P)=F_{Y}(P) Y_{p}(P)
$$

The two legs of the latitude survey of 2009 present distinguishable solar modulation conditions and provide two estimates of the yield functions. For the southbound interval, we use the solar modulation parameter determined using the time dependent PAMELA spectrum [16] [and references therein]. The average modulation parameter estimated from the PAMELA monthly spectra from November and December 2009 is $\Phi_{P A M, S B}=414 \pm 30$ MV (private communication with authors of [14-16]. To the best of the authors' knowledge, time dependent spectra from PAMELA have not been published for the northbound interval. Therefore we use the time dependent solar modulation parameter calculated from the McMurdo neutron monitor counting rate [16, 18]. During the northbound interval the average value was $\Phi_{M c M, N B}=484 \mathrm{MV}$ with a standard deviation of $19 \mathrm{MV}$. (Data were extracted from the database located at http://lpsc.in2p3.fr/crdb/). As a consistency check, during the Southbound interval, the average hourly solar modulation parameter was $\Phi_{M c M, S B}=422$ $\mathrm{MV}$ with a standard deviation of $8 \mathrm{MV}$, in agreement with $\Phi_{P A M, S B}=414 \pm 30 \mathrm{MV}$.

With this set of assumptions, and using $F_{h n, G}$, the yield functions were calculated numerically and are presented in Figure 4(a). There is a very good agreement between the yield functions calculated for the two periods, with a ratio compatible with 1 within the uncertainty (bands in Figure $4(\mathrm{~b})$ represent $1 \sigma$ statistical uncertainty). Changing $F_{h n}$ to $F_{h n, C}$ would induce a systematic change of about $3 \%$ to the yield functions above $3 \mathrm{GV}$. Below $3 \mathrm{GV}$, the statistical uncertainties dominate and the results are not sensitive to the choice between the two factors.

\section{Conclusions}

We performed a latitude survey of two bare neutron counters with compact paraffin moderators that detected atmospheric showers from galactic cosmic rays on board the Swedish icebreaker 
Oden during 2009-2010. By taking such measurements as the ship traversed a wide range of magnetic latitudes, we were able to measure the response function of the bare detectors for the distinct conditions of solar modulation (i.e., effects of solar activity on the galactic cosmic ray flux) during the southbound interval (mostly late 2009) and the northbound interval (early 2010). Combining these measurements with cosmic ray spectra inferred from the PAMELA spacecraft, we derived two estimates of the yield function that are in very good agreement, marking the first direct determination of the yield function of a specific configuration of bare neutron detectors.

The research is supported in part by Thailand Science Research and Innovation via Research Team Promotion Grant RTA6280002.

\section{References}

[1] Hatton, C. J. \& H. Carmichael (1964), Can. J. Phys., 42, 2443-2472

[2] Villoresi, G., L. I. Dorman, N. Iucci et al (2000), J. Geophys. Res. Space Phys., 105, $21025-$ 21034

[3] Nuntiyakul, W., A. Sáiz, D. Ruffolo et al. (2018), J. Geophys. Res. Space Phys., 123, 71817195

[4] Mishev, A.L., I. G. Usoskin, \& G. A. Kovaltsov (2013), J. Geophys. Res. Space Phys., 118, $2783-2788$

[5] Stoker, P. H. (1985), in Proc. $19^{\text {th }}$ ICRC (La Jolla), 4, 114-117

[6] Nuntiyakul, W., P. Evenson, D. Ruffolo et al. (2014), Astrophys J., 795, 11

[7] Potgieter, M. S., B. C. Raubenheimer, P. H. Stoker et al (1980), S. Afr. J. Phys., 3(3-4), 77-89

[8] Pyle, R., P. Evenson, J.W. Bieber et al. (1999), in Proc. 26 ${ }^{\text {th }}$ ICRC (Salt Lake City), SH 3.6.26

[9] Abbasi, R., Y. Abdou, M. Ackermann et al. (2013). Nucl. Instrum. Methods Phys. Res. A, $700,188-220$

[10] Lin, Z., J. W. Bieber, \& P. Evenson (1995), J. Geophys. Res. Space Phys., 100, 23543 - 23550

[11] Bieber, J. W., J. Clem, \& P. Evenson (1997), in Proc. 12 ${ }^{\text {th }}$ ICRC (Hobart), 2, 389-392

[12] Clem, J. M., J. W. Bieber, P. Evenson et al. (1997), J. Geophys. Res., 102, 26, 919-26

[13] Caballero-Lopez, R. A., \& H. Moraal (2012), J. Geophys. Res., 117, A12103

[14] Ghelfi, A., F. Barao, L. Derome, \& D. Maurin (2016), Astron. Astrophys., 591, A94

[15] Ghelfi, A., F. Barao, L. Derome, \& D. Maurin (2017a), Astron. Astrophys., 605, C2

[16] Ghelfi, A., D. Maurin, A. Cheminet et al. (2017b), Adv. in Space Res., 60, 833-847

[17] Gleeson, L. J., \& W. I. Axford (1968), Astrophys J., 154, 1011-1026

[18] Maurin, D., F. Melot, \& R. Taillet (2014), Astron. \& Astrophys., 569, A32 